\title{
Two Cases of Acute Exacerbation of Interstitial Pneumonia Treated with Polymyxin B-immobilized Fiber Column Hemoperfusion Treatment
}

\author{
Satoshi Noma ${ }^{1}$, Wataru Matsuyama ${ }^{1}$, Hideo Mitsuyama ${ }^{1}$, Takayuki Suetsugu ${ }^{1}$, \\ Yoshimizu Koreeda ${ }^{1}$, Keiko Mizuno ${ }^{1}$, Ikkou Higashimoto ${ }^{1}$, Yasuyuki Kakihana ${ }^{2}$, \\ Teruto Hashiguchi ${ }^{3}$, Ikuro Maruyama ${ }^{3}$, Mitsuhiro Osame ${ }^{1}$ and Kimiyoshi Arimura ${ }^{1}$
}

\begin{abstract}
The effect of polymixin B-immobilized fiber column (PMX) hemoperfusion treatment for acute exacerbation of interstitial pneumonia (IP) has been reported. Here, we report 2 cases of acute exacerbation of IP successfully treated with PMX hemoperfusion. One is a 55-year-old woman who was diagnosed as microscopic polyangiitis (MPA) with IP. The other is a 58-year-old man, diagnosed as having idiopathic pulmonary fibrosis. Both cases were treated with PMX hemoperfusion and other therapies. One died on day 44 and the other is still alive. The PMX hemoperfusion treatment decreased the serum levels of several cytokines and activated neutrophil percentage in bronchoalveolar lavage fluid.
\end{abstract}

Key words: bronchoalveolar lavage fluid cells, CD18+/CD11c, high mobility group box-1 (HMGB-1)

(DOI: 10.2169/internalmedicine.46.0117)

\section{Introduction}

Acute exacerbation of idiopathic pulmonary fibrosis (IPF) is a well-recognized concept (1-3) and that of other IPs, in particular those related to collagen vascular diseases have been also reported $(4,5)$. However, effective treatments are not well-established and mortality of these patients has been reported to be high during the short course $(3,6,7)$. Recent reports have suggested that the use of polymixin Bimmobilized fiber column (PMX) hemoperfusion treatment may be effective in patients with acute lung injury (ALI)/ acute respiratory distress syndrome (ARDS) and acute exacerbation of idiopathic pulmonary fibrosis(IPF) (8-11). Here, we report two cases of acute exacerbation of IP treated with PMX hemoperfusion treatment. We also show the flowcytometry results of bronchial lavage fluid (BALF) cells and change of serum cytokine levels before and after PMX hemoperfusion.

\section{Case Report}

\section{Case 1}

A 55-year-old woman was admitted to our hospital in June 2006 because of dyspnea and cough. One year before admission, she was diagnosed as having microscopic polyangiitis with interstitial pneumonia because renal biopsy showed crescentic necrotizing glomerulonephritis and her serum myeloperoxidase antineutrophil cytoplasmic autoantibody (MPO-ANCA) by enzyme-linked immunosorbent assay (ELISA) was very high $(1,937 \mathrm{U} / \mathrm{ml}$, normal range, <9). The chest X-ray and chest high-resolution computed tomography (HRCT) scans showed reticular shadows, ground glass opacities and peripheral subpleural thickening. Her pulmonary symptoms and renal malfunction was improved via the treatment with prednisolone and cyclophosphamide. Three days before admission she developed dyspnea on ef-

\footnotetext{
${ }^{1}$ Division of Respiratory Medicine, Respiratory and Stress Care Center, Kagoshima University Hospital, Kagoshima, ${ }^{2}$ Division of Intensive Care Medicine, Kagoshima University Hospital, Kagoshima and ${ }^{3}$ Department of Laboratory and Vascular Medicine Cardiovascular and Respiratory Disorders Advanced Therapeutics, Kagoshima University Graduate School of Medical and Dental Science, Kagoshima

Received for publication February 22, 2007; Accepted for publication May 21, 2007

Correspondence to Dr. Wataru Matsuyama, vega@xa2.so-net.ne.jp
} 


\section{Before PMX treatment}
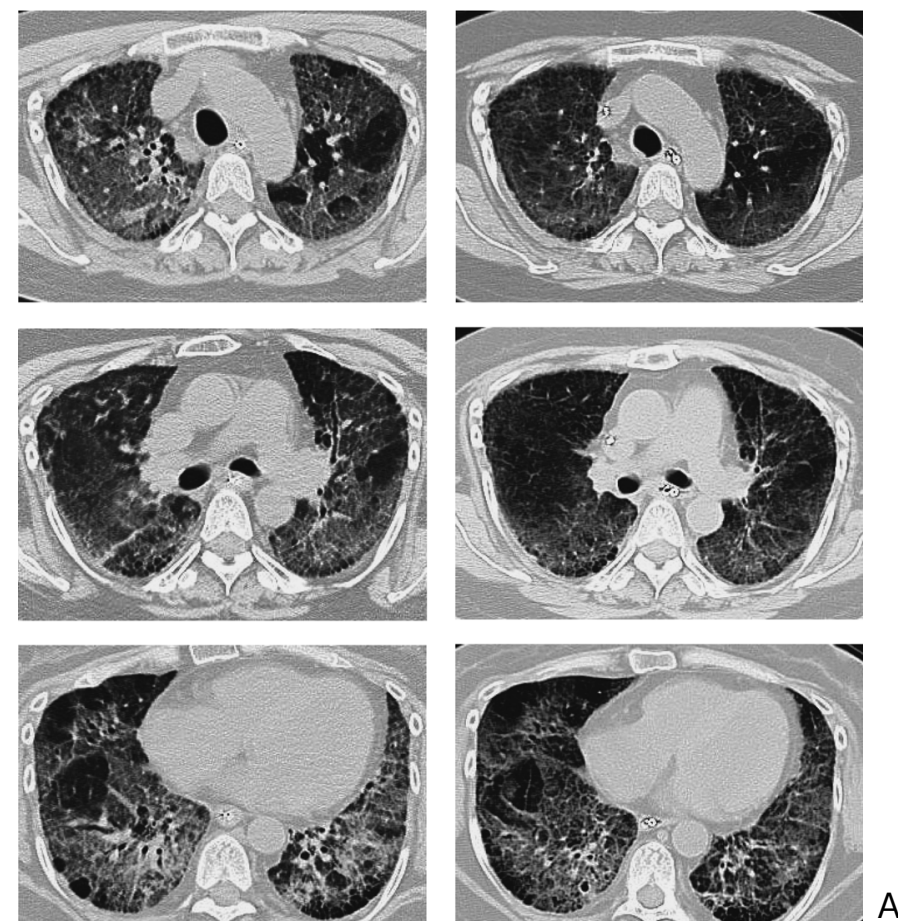
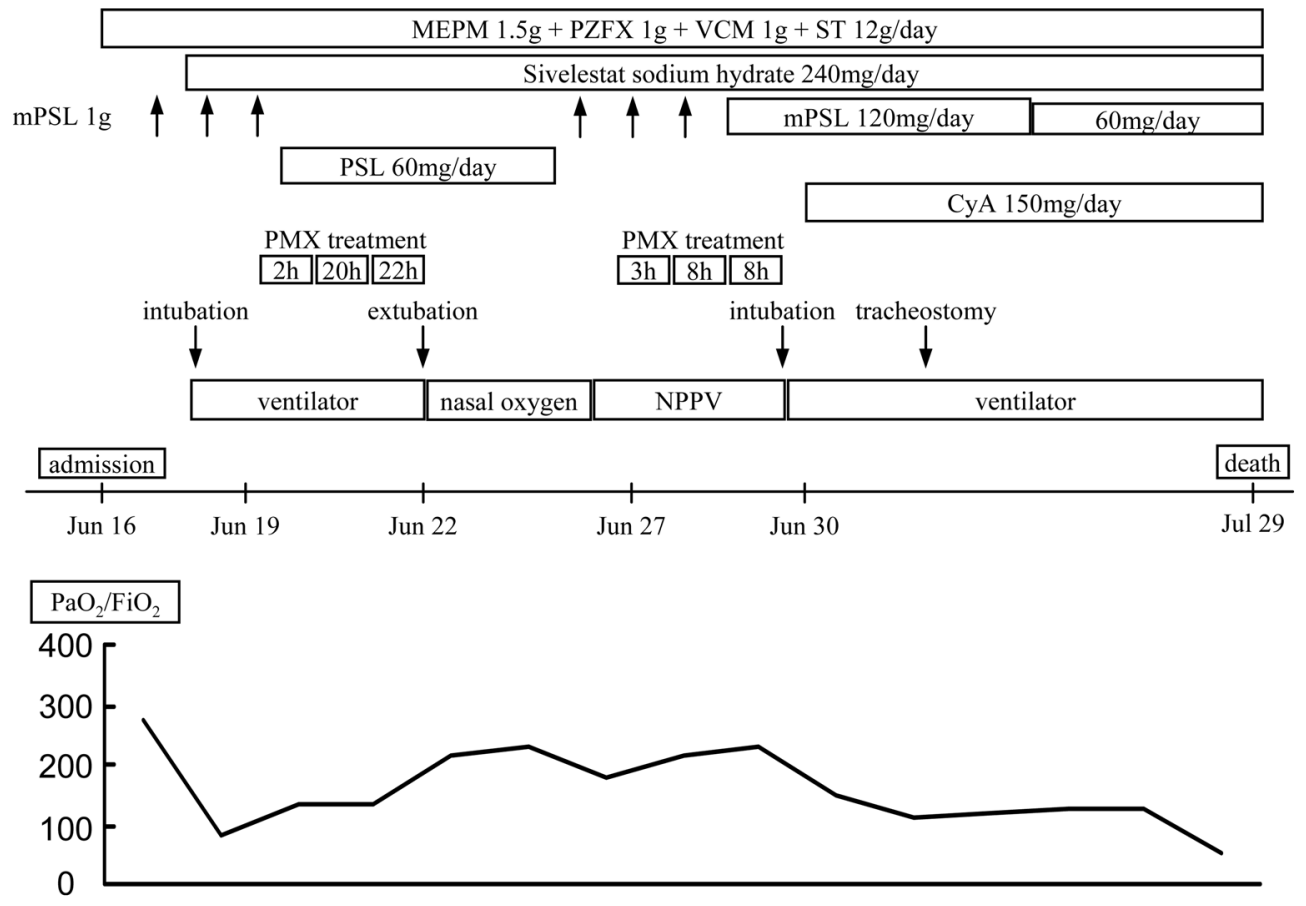

B

Figure 1. A: Chest HRCT before and after the PMX hemoperfusion treatment of Case 1. Chest HRCT showed improvement of the ground-glass opacity. B: Clinical course of Case 1.

fort and cough. Her symptoms became worse and she was admitted to our hospital. Laboratory findings on admission were as follows: white blood cell count (WBC) $9,700 \mathrm{~mm}^{3}$; lactate dehydrogenase $(\mathrm{LDH}) 543 \mathrm{IU} / \mathrm{L}$; C-reactive protein (CRP) $7.43 \mathrm{mg} / \mathrm{dl}$; KL-6 3,373 U/mL. Endotoxin and $\beta$-D glucan concentrations were under the detectable limits. MPO-ANCA was 22.0 U/ml. Arterial blood gas analysis under room air showed hypoxia and hypocarbia $(\mathrm{pH} 7.512$, $\mathrm{PaCO}_{2} 33.3 \mathrm{mmHg}, \mathrm{PaO}_{2} 57.2 \mathrm{mmHg}$ ). The chest HRCT scans showed diffuse bilateral ground glass opacities (Fig. 1A). The BALF anlaysis showed an increase in neutrophils with no obvious evidence of infectious disease (Table 1). Her clinical course is shown in Fig. 1B. She was treated with steroid pulse therapy ( $1 \mathrm{~g}$ of methylpredonisolone per day for 3 days), Sivelestat sodium hydrate, and antibiotics. However, the respiratory failure progressed and mechanical ventilation was applied on day 3 after admission. On day 4 we started PMX hemoperfusion treatment 
Table 1. BALF Finding

\begin{tabular}{|c|c|c|}
\hline & Before PMX & After PMX \\
\hline \multicolumn{3}{|l|}{ Case 1} \\
\hline Total cell count $(/ \mu \mathrm{l})$ & $6.41 \times 10^{5}$ & $1.57 \times 10^{5}$ \\
\hline Macrophage $(\%)$ & 43 & 76 \\
\hline Lymphocyte (\%) & 16 & 11 \\
\hline Neutrophil (\%) & 41 & 12 \\
\hline Eosinophil (\%) & 1 & 1 \\
\hline $\mathrm{CD} 4 / 8$ & 0.89 & 1.02 \\
\hline $\mathrm{CD} 18+/ \mathrm{CD} 11 \mathrm{c}+(\%)$ & 34.3 & 11.2 \\
\hline CXCR2+ $(\%)$ & 18.2 & 9.1 \\
\hline CCR2+(\%) & 18.4 & 9.3 \\
\hline $\mathrm{CD} 3+/ \mathrm{CD} 25+(\%)$ & 21.8 & 22.1 \\
\hline CXCR3+ $(\%)$ & 9.2 & 9.1 \\
\hline Bacteria & $(-)$ & $(-)$ \\
\hline PJ-PCR & $(-)$ & $(-)$ \\
\hline \multicolumn{3}{|l|}{ Case 2} \\
\hline Total cell count $(/ \mu \mathrm{l})$ & $0.9 \times 10^{5}$ & $0.53 \times 10^{5}$ \\
\hline Macrophage $(\%)$ & 64 & 44 \\
\hline Lymphocyte (\%) & 16 & 17 \\
\hline Neutrophil (\%) & 16 & 36 \\
\hline Eosinophil (\%) & 3 & 3 \\
\hline $\mathrm{CD} 4 / 8$ & 2.6 & 1.7 \\
\hline $\mathrm{CD} 18+/ \mathrm{CD} 11 \mathrm{c}+(\%)$ & 13.1 & 9.2 \\
\hline CXCR2+ $(\%)$ & 9.2 & 4.1 \\
\hline CCR2+ (\%) & 13.3 & 6.3 \\
\hline $\mathrm{CD} 3+/ \mathrm{CD} 25+(\%)$ & 14.1 & 15.6 \\
\hline CXCR3+ $(\%)$ & 7.2 & 7.4 \\
\hline Bacteria & $(-)$ & $(-)$ \\
\hline PJ-PCR & $(-)$ & $(-)$ \\
\hline
\end{tabular}

(Toraymixin 20R, Toray Medical Co., Tokyo, Japan, a flow rate of $80 \mathrm{ml} / \mathrm{min}$ ). Nafamostat mesilate (Torii Pharma Co., Tokyo, Japan) was used as an anticoagulant during the PMX treatment. We performed PMX hemoperfusion treatment 3 times ( 2 hours, 22 hours and 20 hours). After treatment, the $\mathrm{PaO}_{2} / \mathrm{FiO}_{2}$ (P/F) ratio improved from 64.2 to 229.4 , and she was successfully weaned from mechanical ventilation. Chest HRCT showed improvement of the ground-glass opacity (Fig. 1A). The laboratory findings at this point were as follows: lactate dehydrogenase (LDH) 243 IU/L; C-reactive protein (CRP) $0.43 \mathrm{mg} / \mathrm{dl}$; KL-6 2,139 U/mL. Endotoxin and $\beta$-D glucan concentrations were under the detectable limits. MPO-ANCA was $7.3 \mathrm{U} / \mathrm{ml}$. BALF after treatment showed decreased total cell counts and neutrophil percentage. Twelve days after the remission, she again developed respiratory failure and we applied mechanical ventilation. The respiratory failure progressed despite the therapy including PMX hemoperfusion treatment and she died because of respiratory failure 44 days after admission (41 days after the first PMX hemoperfusion treatment. An autopsy was performed, which showed diffuse alveolar damage with hyaline membranes and partial organization. Acute bronchopneumonia was found in the right lower lobe.

\section{Case 2}

A 58-year-old man was admitted to our hospital because of dyspnea in December 2006. Three years before admission, health examination showed an abnormal chest shadow, but he did not go to the hospital. Eight months before admission, he came to our hospital due to dyspnea on effort. His chest HRCT revealed reticular opacities, honeycombing and traction bronchiectasis with basal and peripheral predominance. There was no evidence of vascular collagen disease, hypersensitivity pneumonitis, pneumoconiosis, sarcoidosis, or vasculitis. He was diagnosed as having IPF clinically according to the criteria of American Thoracic Society/ European Respiratory Society (ATS/ERS) international consensus statement (12). His mother had also suffered from interstitial pneumonia and died because of respiratory failure. Five days before admission, he developed dyspnea on effort (Hugh-Jones dyspnea criteria grade II) and cough. The symptoms became worse (Hugh-Jones dyspnea criteria grade IV) and he was admitted to our hospital. Laboratory findings on admission were as follows: WBC 7,700 $\mathrm{mm}^{3}$; $\mathrm{LDH}$ 348 IU/L; CRP 8.32 mg/dl; KL-6 1,548 U/mL. Endotoxin and $\beta$-D glucan concentrations were under the detectable limits. Arterial blood gas analysis under room air showed hypoxia ( $\mathrm{pH} 7.425, \mathrm{PCO}_{2} 38.5 \mathrm{mmHg}, \mathrm{PO}_{2} 36.1 \mathrm{mmHg}$ ). Chest HRCT showed diffuse bilateral ground glass opacities predominantly in lower lobe (Fig. 2A). BALF examination showed an increase of neutrophil percentage with no evidence of infection. His clinical course is shown in Fig. 2B. We started antibiotics, steroid pulse therapy ( $1 \mathrm{~g}$ of methylprednisolone per day for 3 days), cyclophosphamide pulse therapy (500 mg of cyclophosphamide per day), and antibiotic therapy. On day 2 after admission, we started PMX he- 
Before PMX treatment After PMX treatment
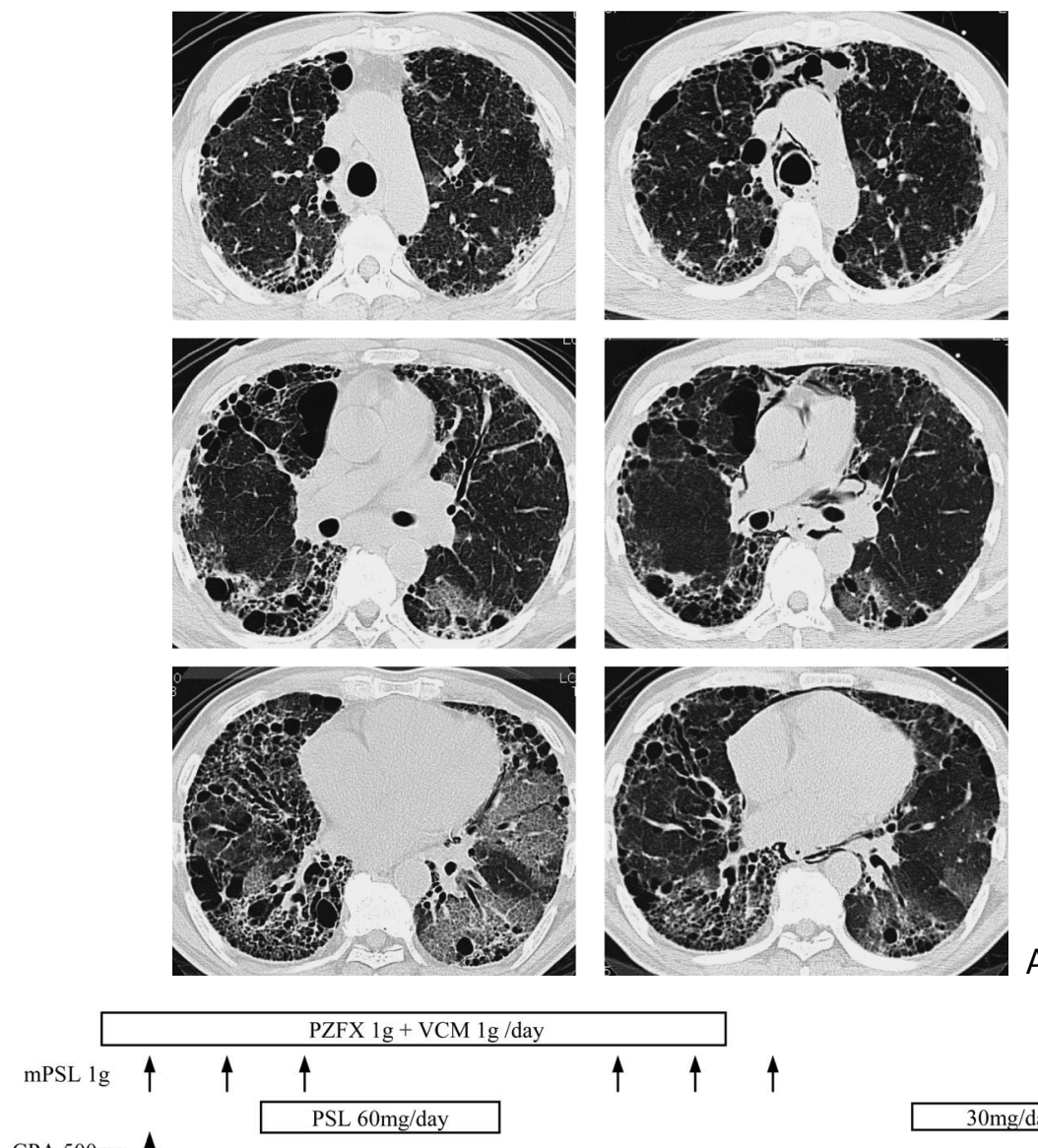

CPA 500mg $\uparrow$

PSL 60mg/day

$30 \mathrm{mg} /$ day

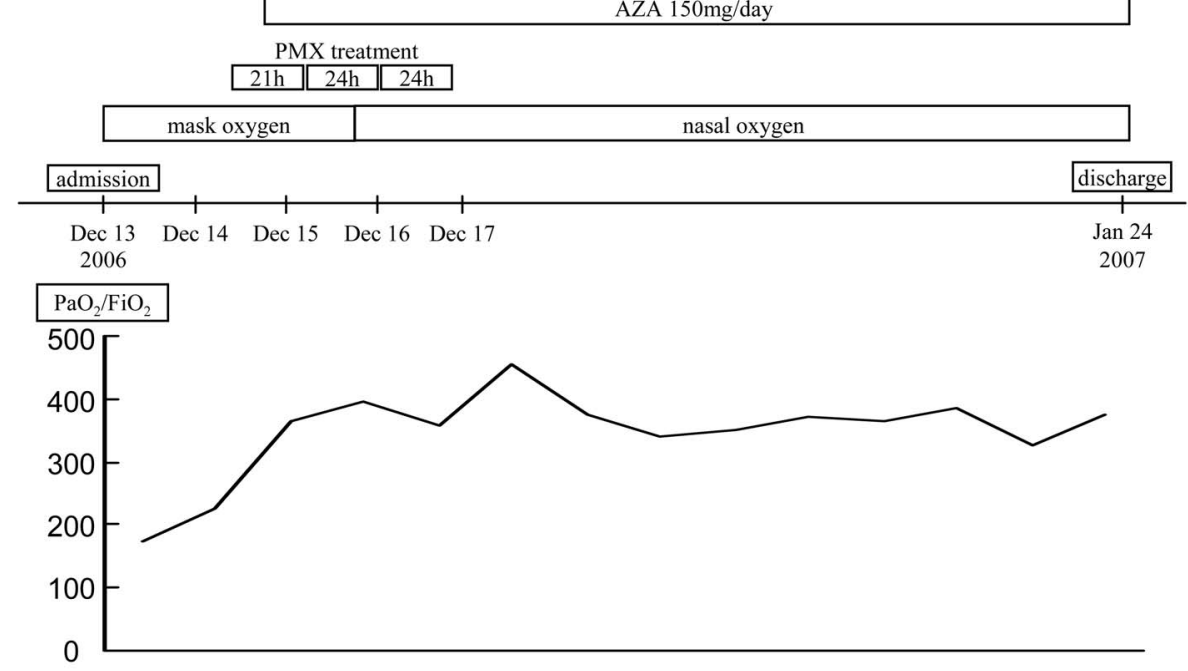

Figure 2. A: Chest HRCT before and after the PMX hemoperfusion treatment of Case 2. Chest HRCT showed improvement of the ground-glass opacity. B: Clinical course of Case 2.

moperfusion treatment (Toraymixin 20R, Toray Medical Co., Tokyo, Japan, at a flow rate of $80 \mathrm{ml} / \mathrm{min}$ ). Nafamostat mesilate (Torii Pharma Co., Tokyo, Japan) was used as an anticoagulant during the PMX hemoperfusion treatment. We performed PMX hemoperfusion treatment 3 times (20 hours, 24 hours and 24 hours). After PMX hemoperfusion treatment, $\mathrm{P} / \mathrm{F}$ ratio improved from 225.0 to 395.6. Chest HRCT showed improvement of the ground-glass opacity (Fig. 2A).
The laboratory findings at this point were as follows: LDH $221 \mathrm{IU} / \mathrm{L}$; CRP $0.32 \mathrm{mg} / \mathrm{dl}$; KL-6 $748 \mathrm{U} / \mathrm{mL}$; endotoxin and $\beta$-D glucan were under detectable concentration. BALF examination after PMX hemoperfusion treatment showed decreased total cell counts and an increase of neutrophil percentage. Prednisolone (30 mg/day) and azathioprine (150 $\mathrm{mg} /$ day) was administered orally as maintenance therapy. His symptoms improved and he was discharged 44 days af- 


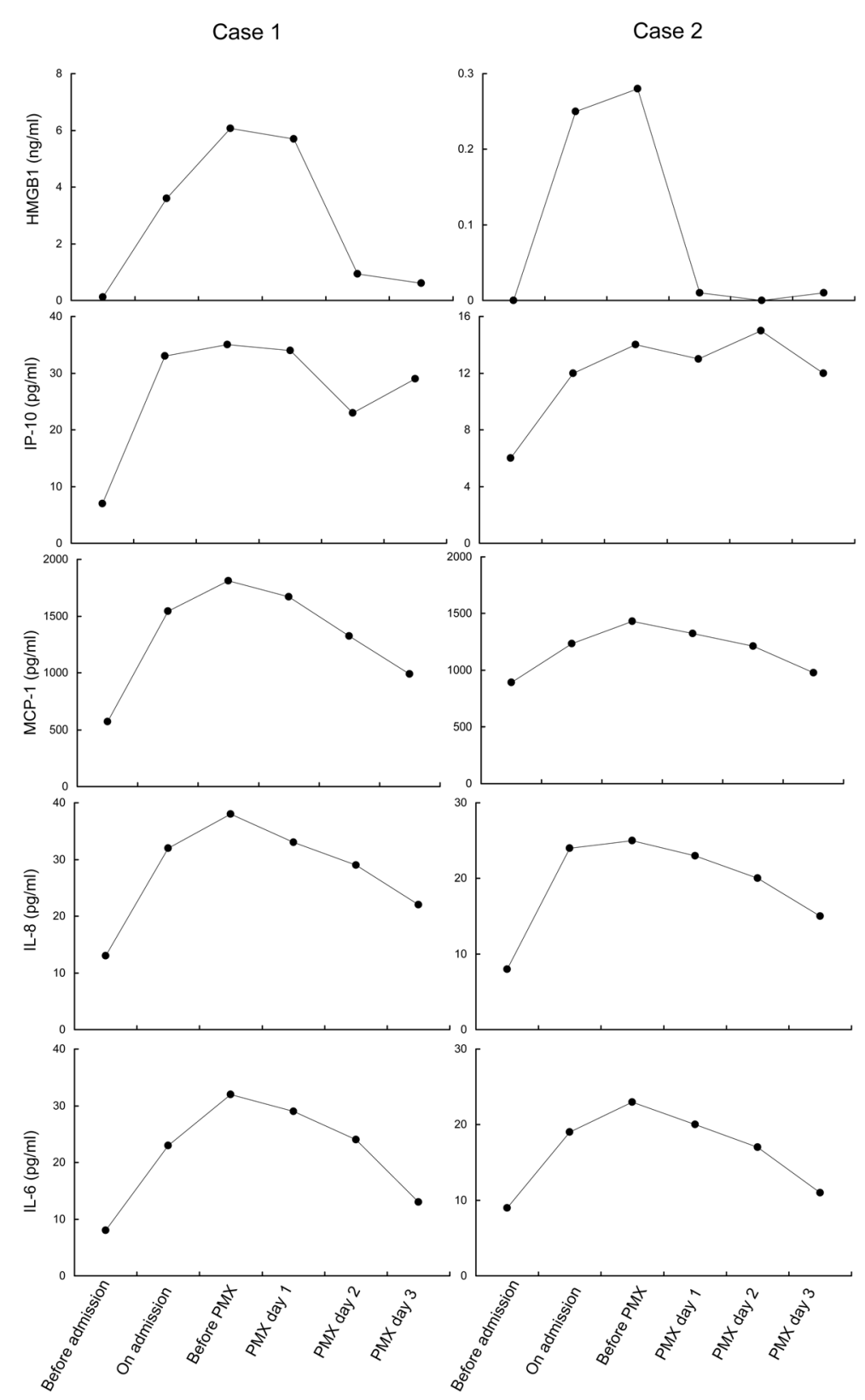

Figure 3. Cytokine changes in sera of our cases during the PMX hemoperfusion treatment. The serum HMGB1 level was undetectable before admission, increased on admission, and decreased remarkably on day 2 after the start of PMX hemoperfusion treatment in both cases. The serum level of IP-10 did not show remarkable change during the PMX hemoperfusion treatment. The serum level of MCP-1, IL-8, and IL-6 decreased on day 3 after the start of PMX hemoperfusion treatment, but was higher than before admission in both cases.

ter admission.

\section{Criteria for the PMX hemoperfusion treatment on acute exacerbation of interstitial pneumonia}

As a policy of our department, we administrate PMX hemoperfusion to the patients who fulfill the following criteria: 1) acute exacerbation of interstitial pneumonia; 2) no obvious evidence of infectious disease; 3) P/F ratio does not increase above 300 (criteria for acute lung injury (13)) within 24 hours despite the start of standard treatments for acute exacerbation of interstitial pneumonia.

\section{Measurement of serum cytokine level}

We measured interleukin-6 (IL-6), IL-8, monocyte chemoattractant protein-1 (MCP-1) and interferon inducible protein of $10 \mathrm{kDa}$ (IP-10) levels in sera of our patients using a commercial enzyme-linked immunosorbent assay (ELISA) kit (sIL-2R: R\&D Systems, Minneapolis, MN). ELISA for high mobility group box 1 (HMGB1) in the sera was performed with the use of monoclonal antibodies to HMGB1 and with standardization to a curve of recombinant human HMGB1 as described previously (14). As shown in Fig. 3, The serum HMGB1 level was undetectable before admission, increased on admission, and decreased remarkably on day 2 after the 
start of PMX hemoperfusion treatment in both cases. The serum level of IP-10 did not show a remarkable change during the PMX hemoperfusion treatment. The serum levels of MCP-1, IL-8, and IL-6 decreased on day 3 after the start of PMX hemoperfusion treatment, but were higher than before admission in both cases.

\section{Flow cytometry analysis of BALF cells}

The expression of BALF cell surface CD3, CD18, CD11c, CD25 (interleukin-2 receptor), CXCR2 (receptor of IL-8), CXCR3 (receptor for IP-10) and CCR2 (receptor of MCP-1) (15) was evaluated by flow-cytometry analysis. One hundred thousand BALF cells were suspended in $50 \mu \mathrm{l}$ of cold PBS containing $0.1 \%$ sodium azide, $10 \mathrm{ng} / \mathrm{ml}$ BSA and $20 \mu \mathrm{g} / \mathrm{ml}$ of human IgG, incubated for 10 minutes on ice, and with mouse monoclonal anti-CXCR2, CD18, CCR2, CXCR3 or CD25 (PharMingen, SanDiego, CA, USA) IgG antibody and FITC-conjugated anti-CD3 or PE-conjugated CD11c antibody (PharMingen) for an additional 15 minutes on ice. Cells were washed with PBS, and incubated with FITC or PE-conjugated goat anti-mouse IgG for 15 minutes on ice. At the end of the incubation, 7AAD (PharMingen) was added to each tube. The cells were washed with PBS, and subsequently analyzed by flow cytometry using a FACScan (Becton Dickinson). Dead cells, determined by the incorporation of 7AAD, were gated out. Results were processed using the CellQuest software (Becton Dickson).

As shown in Table 1, the percentage of CD18+/CD11c+, $\mathrm{CCR} 2+$, and/CXCR2+BALF cell decreased remarkably while $\mathrm{CD} 3+/ \mathrm{CD} 25+$ and CXCR3+ BALF cell percentage did not show remarkable change.

\section{Discussion}

Acute exacerbations of IPs have been described to have high mortality during short course $(3,6,7)$. Recent reports have suggested that PMX hemoperfusion treatment may be effective in patients with ALI/ARDS and acute exacerbation of IPF $(8,9,11)$. PMX hemoperfusion treatment can reduce the mortality of ARDS (11) and reduce serum level of metalloproteinase (MMP)-9 tissue inhibitor of MMP (TIMP)-1 (9). Also, PMX hemoperfusion treatment can improve the survival rate of sepsis with renal failure (16) and oxygenation of sepsis patients (17). In particular, Seo et al reported that four of six patients of acute exacerbations of IPF were successfully weaned from mechanical ventilation and survived more than 30 days after the initial PMX hemoperfusion treatment, suggesting the clinical effect of PMX hemoperfusion treatment (10). The present cases also survived more than 30 days, however, the outcome of our two cases was totally different (case 1 died while case 2 is still alive on April 25, 2007). We think this difference might have been due to the following reasons: 1) The difference of timing of PMX hemoperfusion administration [Early administration of PMX hemoperfusion treatment can eliminate humoral mediators and improves pulmonary oxygenation.]. (8); 2) The presence of infection in case 1 (Case 1 showed bronchopneumonia at autopsy). However, it is difficult to determine the true reason for this difference. Detection of the true reason of this difference might provide an important insight to clarify the mechanism of PMX treatment in acute exacerbation of interstitial pneumonia.

The detailed mechanism by which PMX hemoperfusion treatment improves oxygenation has not been fully elucidated. In the present cases, we showed a decrease of IL-8, MCP-1, IL-6, and HMGB1 level in the sera, however, the serum IP-10 level did not change remarkably during the PMX treatment. IL-8, a chemokine that can activate neutrophils through CXCR2 (15), is elevated in IPF and indicates disease activity (18) and is associated with the pathogenesis of MPO-ANCA vasculitis (19). MCP-1, a chemokine that can attract a variety of inflammatory cells including monocytes and neutrophils (15), is a marker of ANCA-associated vasculitis (20) and is involved in the pathogenesis of IPF (21). PMX hemoperfusion treatment can reduce the serum level of IL-6 and HMGB1 (22). IL-6 is an important inflammatory mediator (23). HMGB-1 has recently been proposed as one of the late mediators of sepsis or lipopolysaccharide (LPS) endotoxin lethality (24). Abraham et al demonstrated that the intratracheal administration of recombinant HMGB1 is a distal mediator of acute inflammatory lung injury (25). Ueno et al suggested that the overexpression of extracellular HMGB-1 plays a key role in the pathogenesis of ALI (26). The modification of these cytokines might be associated with the effect of PMX hemoperfusion treatment on acute exacerbation of interstitial pneumonia.

In the present cases, CD18+/CD11c+ and CXCR2+ BALF cell percentage decreased after the PMX hemoperfusion treatment. CD18+/CD11c+ is a marker of activated neutrophils (27), and CXCR2 is a receptor of IL-8 (15). In our report, we found that a decrease in cytokine is associated with activation of neutrophils. This change might contribute to the decrease of CD18+CD11c positive cells. However, the reason for the increase in neutrophil $\%$ after the PMX treatment in case 2 is still unclear. Ambrosini et al reported that in acute exacerbations of IPF, marked neutrophilia was detected in BALF (3). Neutrophils cause damage to the pulmonary vascular endothelium by releasing oxygen radicals, proteinases, leukotrienes, and other proinflammatory molecules such as platelet-activating factor, thus impairing the barrier function of the pulmonary capillaries and leading to the onset of ALI or ARDS $(28,29)$. In our cases, CCR2+ BALF cell percentage decreased after the PMX hemoperfusion treatment. MCP-1-CCR2 axis contributes to the neutrophil recruitment in lung via alveolar macrophages in lung inflammation (30). In the present cases, CD3+/CD25+ BALF cell percentage and CXCR3 (receptor for of IP-10 (15)) positive cell percentage did not show remarkable change. $\mathrm{CD} 3+/ \mathrm{CD} 25+$ cells are activated $\mathrm{T}$ lymphocytes (31), and IP-10 is a chemokine that can recruit activated T lymphocytes (32). These results may suggest the relation between the effect of PMX hemoperfusion treatment and acti- 
vated neutrophils. In summary, we reported two cases of acute exacerbation of interstitial pneumonia who received polymyxin B-immobilized fiber column hemoperfusion treatment. Also, our report showed flow-cytometry analysis of BALF cells and a change in the serum HMGB1 level that was not investigated in previous reports. Further accumulation of clinical data concerning this point might bring new insight to clarify the detailed mechanism by which PMX hemoperfusion treatment improves oxygenation.

We wish special thanks to Mrs. Rumi Matsuyama (Third Department of Internal Medicine, Kagoshima University Faculty of Medicine) for her excellent help.

\section{References}

1. Kondoh Y, Taniguchi H, Kawabata Y, Yokoi T, Suzuki K, Takagi $\mathrm{K}$. Acute exacerbation in idiopathic pulmonary fibrosis. Analysis of clinical and pathologic findings in three cases. Chest 103: 1808-1812, 1993

2. Parambil JG, Myers JL, Ryu JH. Histopathologic features and outcome of patients with acute exacerbation of idiopathic pulmonary fibrosis undergoing surgical lung biopsy. Chest 128: 3310-3315, 2005

3. Ambrosini V, Cancellieri A, Chilosi M, et al. Acute exacerbation of idiopathic pulmonary fibrosis: report of a series. Eur Respir J 22: 821-826, 2003

4. Parambil JG, Myers JL, Ryu JH. Diffuse alveolar damage: uncommon manifestation of pulmonary involvement in patients with connective tissue diseases. Chest 130: 553-558, 2006.

5. Parambil JG, Myers JL, Lindell RM, Matteson EL, Ryu JH. Interstitial lung disease in primary Sjögren syndrome. Chest 130: 1489-1495, 2006.

6. Akira M, Hamada H, Sakatani M, Kobayashi C, Nishioka M, Yamamoto S. CT findings during phase of accelerated deterioration in patients with idiopathic pulmonary fibrosis. AJR Am J Roentgenol 168: 79-83, 1997.

7. Rice AJ, Wells AU, Bouros D, et al. Terminal diffuse alveolar damage in relation to interstitial pneumonias. An autopsy study. Am J Clin Pathol 119: 709-714, 2003.

8. Kushi H, Miki T, Okamaoto K, Nakahara J, Saito T, Tanjoh K. Early hemoperfusion with an immobilized polymyxin B fiber column eliminates humoral mediators and improves pulmonary oxygenation. Crit Care 9: R653-R661, 2005.

9. Nakamura T, Kawagoe Y, Matsuda T, et al. Effect of polymyxin B-immobilized fiber on blood metalloproteinase- 9 and tissue inhibitor of metalloproteinase-1 levels in acute respiratory distress syndrome patients. Blood Purif 22: 256-260, 2004.

10. Seo Y, Abe S, Kurahara M, et al. Beneficial effect of polymyxin B-immobilized fiber column (PMX) hemoperfusion treatment on acute exacerbation of idiopathic pulmonary fibrosis. Intern Med 45: 1033-1038, 2006

11. Tsushima K, Kubo K, Koizumi T, et al. Direct hemoperfusion using a polymyxin $\mathrm{B}$ immobilized column improves acute respiratory distress syndrome. J Clin Apher 17: 97-102, 2002.

12. American Thoracic Society/European Respiratory Society International Multidisciplinary Consensus Classification of the Idiopathic Interstitial Pneumonias. This joint statement of the American Thoracic Society (ATS), and the European Respiratory Society (ERS) was adopted by the ATS board of directors, June 2001 and by the ERS Executive Committee, June 2001. Am J Respir Crit Care Med 165: 277-304, 2002.

13. Bernard GR, Artigas A, Brigham KL, et al. The AmericanEuropean Consensus Conference on ARDS. Definitions, mechanisms, relevant outcomes, and clinical trial coordination. Am J Respir Crit Care Med 149: 818-824, 1994.

14. Yamada S, Inoue K, Yakabe K, Imaizumi H, Maruyama I. High mobility group protein 1 (HMGB1) quantified by ELISA with a monoclonal antibody that does not cross-react with HMGB2. Clin Chem 49: 1535-1537, 2003.
15. Rollins BJ. Chemokines. Blood 90: 909-928, 1997.

16. Suzuki $H$, Nemoto $H$, Nakamoto $H$, et al. Continuous hemodiafiltration with polymyxin-B immobilized fiber is effective in patients with sepsis syndrome and acute renal failure. Ther Apher 6: 234240, 2002.

17. Naka T, Shinozaki M, Akizawa T, Shima Y, Takaesu H, Nasu H. The effect of continuous veno-venous hemofiltration or direct hemoperfusion with polymyxin B-immobilized fiber on neutrophil respiratory oxidative burst in patients with sepsis and septic shock. Ther Apher Dial 10: 7-11, 2006.

18. Ziegenhagen MW, Zabel P, Zissel G, Schlaak M, MullerQuernheim J. Serum level of interleukin 8 is elevated in idiopathic pulmonary fibrosis and indicates disease activity. Am J Respir Crit Care Med 157: 762-768, 1998.

19. Hsieh SC, Yu HS, Cheng SH, et al. Anti-myeloperoxidase antibodies enhance phagocytosis, IL-8 production, and glucose uptake of polymorphonuclear neutrophils rather than anti-proteinase 3 antibodies leading to activation-induced cell death of the neutrophils. Clin Rheumatol 26: 216-224, 2007.

20. Tam FW, Sanders JS, George A, et al. Urinary monocyte chemoattractant protein-1 (MCP-1) is a marker of active renal vasculitis. Nephrol Dial Transplant 19: 2761-2768, 2004.

21. Matsuyama W, Watanabe M, Shirahama Y, et al. Activation of discoidin domain receptor 1 on CD14-positive bronchoalveolar lavage fluid cells induces chemokine production in idiopathic pulmonary fibrosis. J Immunol 174: 6490-6498, 2005.

22. Sakamoto Y, Mashiko K, Matsumoto H, et al. Effect of direct hemoperfusion with a polymyxin B immobilized fiber column on high mobility group box-1 (HMGB-1) in severe septic shock: report of a case. ASAIO J 52: e37-e39, 2006.

23. Gabay C. Interleukin- 6 and chronic inflammation. Arthritis Res Ther 8 (Suppl 2): S3, 2006.

24. Wang $\mathrm{H}$, Bloom $\mathrm{O}$, Zhang $\mathrm{M}$, et al. HMG-1 as a late mediator of endotoxin lethality in mice. Science 285: 248-251, 1999.

25. Abraham E, Arcaroli J, Carmody A, Wang H, Tracey KJ. HMG-1 as a mediator of acute lung inflammation. J Immunol 165: 29502954, 2000.

26. Ueno H, Matsuda $T$, Hashimoto $S$, et al. Contributions of high mobility group box protein in experimental and clinical acute lung injury. Am J Respir Crit Care Med 170: 1310-1316, 2004.

27. Berton G, Laudanna C, Sorio C, Rossi F. Generation of signals activating neutrophil functions by leukocyte integrins: LFA-1 and gp150/95, but not CR3, are able to stimulate the respiratory burst of human neutrophils. J Cell Biol 116: 1007-1017, 1992.

28. Ware LB, Matthay MA. The acute respiratory distress syndrome. N Engl J Med 342: 1334-1349, 2000.

29. Dudek SM, Garcia JG. Cytoskeletal regulation of pulmonary vascular permeability. J Appl Physiol 91: 1487-1500, 2001.

30. Maus UA, Waelsch K, Kuziel WA, et al. Monocytes are potent facilitators of alveolar neutrophil emigration during lung inflammation: role of the CCL2-CCR2 axis. J Immunol 170: 3273-3278, 2003.

31. Smith KA. The interleukin 2 receptor. Annu Rev Cell Biol 5: $397-$ 425, 1989. 
DOI: $10.2169 /$ internalmedicine.46.0117

32. Loetscher M, Gerber B, Loetscher $P$, et al. Chemokine receptor activated T-lymphocytes. J Exp Med 184: 963-969, 1996. specific for IP10 and mig: structure, function, and expression in

(C) 2007 The Japanese Society of Internal Medicine http://www.naika.or.jp/imindex.html 